\begin{tabular}{|c|l|}
\hline Title & $\begin{array}{l}\text { Selective molecular beam epitaxy growth of size and position-control led GaN A I GaN nanowires on nonplanar (0001) } \\
\text { substrates and its growth mechanism }\end{array}$ \\
\hline Author(s) & Sato, Taketomo; Oikawa, Takeshi; Hasegawa, Hideki; Hashizume, Tamotsu \\
\hline Citation & $\begin{array}{l}\text { Journal of V acuum Science \& Technology B Microelectronics and Nanometer Structures, 24(4), 2087-2092 } \\
\text { https://doi.org/10.1116/1.2214700 }\end{array}$ \\
\hline Issue Date & 2006-07-26 \\
\hline Doc URL & http://hdl.handle.net/2115/14595 \\
\hline Type & article \\
\hline File Information & JVSTB.pdf \\
\hline
\end{tabular}

Instructions for use 


\title{
Selective molecular beam epitaxy growth of size- and position-controlled GaN/AIGaN nanowires on nonplanar (0001) substrates and its growth mechanism
}

\author{
Taketomo Sato, ${ }^{\text {a) }}$ Takeshi Oikawa, Hideki Hasegawa, and Tamotsu Hashizume \\ Research Center for Integrated Quantum Electronics (RCIQE), Hokkaido University, North 13, West 8, \\ Kita-Ku, Sapporo 060-8628, Japan and Graduate School of Information Science and Technology, \\ Hokkaido University, North 13, West 8, Kita-Ku, Sapporo 060-8628, Japan
}

(Received 15 January 2006; accepted 3 April 2006; published 26 July 2006)

\begin{abstract}
Fundamental growth properties were investigated for the size-controlled selective MBE growth of $\mathrm{AlGaN} / \mathrm{GaN}$ nanowires on the GaN (0001) prepatterned substrates both experimentally and theoretically. The lateral size of the present GaN nanowire was determined by two facet boundaries formed within AlGaN barrier layers. From the series of wire growth experiments, the growth selectivity and the measured angle of the facet boundary strongly depended on the Al composition and the initial crystalline facets of the mesa patterned templates. The experimental evolution of the cross-sectional structures was well reproduced by a computer simulation based on the phenomenological growth model where the slope angle dependence of lifetime of adatoms was taken into account. The lateral width of present nanowires could be kinetically controlled by the growth conditions and the supply thickness of AlGaN layers. () 2006 American Vacuum Society. [DOI: 10.1116/1.2214700]
\end{abstract}

\section{INTRODUCTION}

In addition to blue/UV photonic and high power electronic device applications, the $\mathrm{AlGaN} / \mathrm{GaN}$ system seems to be potentially useful for the realization of high temperature operating quantum devices, such as quantum wire transistors (QWR-Trs) and single electron transistors (SETs). This is not only due to wide energy gaps with a large $\Delta E_{c}$, but also due to the fact that a high-density two dimensional electron gas (2DEG) is available even without doping which may avoid the doping fluctuation problem in nanostructures.

As for the fabrication technique of quantum nanostructures such as quantum dots (QDs) and QWRs, it is very important to control their size and position precisely as well as to reduce the process-induced damage on the surfaces. The fabrication techniques based on the crystal growth have an advantage as to reduce the processing damage due to the crystal growth nature, as compared with the dry-etching process widely used for the nitride materials. Several groups have reported the growth of $\mathrm{GaN}$ quantum dots using the antisurfactant ${ }^{1,2}$ or Stranski-Krastanov (SK) modes. ${ }^{3,4}$ Intensive research efforts have been also made on the growth of free-standing nanowires and nanocolumns which might be good candidates of the nanomaterials applicable for optoelectronics and nanoelectronics. ${ }^{5,6}$ However, it seems to be extremely difficult to control the position and size of nanostructures simultaneously.

Among the various fabrication techniques of nanostructures, selective growth of III-V semiconductors on prepatterned substrates either by molecular beam epitaxy (MBE) or by metal organic vapor phase epitaxy (MOVPE) is one of the

\footnotetext{
a) Author to whom correspondence should be addressed; electronic mail: taketomo@rciqe.hokudai.ac.jp
}

most promising technique for the formation of size- and position-controlled arrays of quantum nanostructures. ${ }^{7-10}$ Recently, we have reported successful selective MBE growth of InP-based $^{11}$ and GaAs-based ${ }^{12,13}$ QWRs. They included $\langle-110\rangle$-oriented QWRs on (001) substrates and $\langle-1-12\rangle$-oriented wires on (111)B substrates. The minimum wire width was below $10 \mathrm{~nm}$, and the maximum amount of blueshift of the photoluminescence (PL) peak energy due to quantum confinement was $440 \mathrm{meV}$, including a significant contribution from lateral confinement. As for the GaN-based wires, ${ }^{14,15}$ it has shown that the growth of the wire below $40 \mathrm{~nm}$ could be possible on $\langle 11-20\rangle$-oriented mesas patterned on (0001) substrates. Such selective grown wires have the great advantage for the planar integration of quantum devices due to the good controllability of their position as compared with the free-standing nanowires or nanocolumns. However, the growth on nonplanar substrates usually involves various high-index facets simultaneously and this complicates growth kinetics. Especially for GaN-based materials, the most part of their basic growth properties on nonplanar substrates has never been clarified both experimentally and theoretically.

On the basis of our previous work, the purpose of this article is to further elaborate the experimental method and fundamental growth parameters related to size control in the selective MBE growth of $\mathrm{AlGaN} / \mathrm{GaN}$ nanowires by paying attention to the growth mechanism on the nonplanar substrates. In this study, growth of wires was carried out intentionally using large substrate patterns in order to investigate in detail their complex cross-sectional features by scanning electron microscopy (SEM) observation. For the theoretical analysis, a computer simulation method which our group recently developed for GaAs-based materials ${ }^{16,17}$ was first applied in this study for GaN-based materials. 

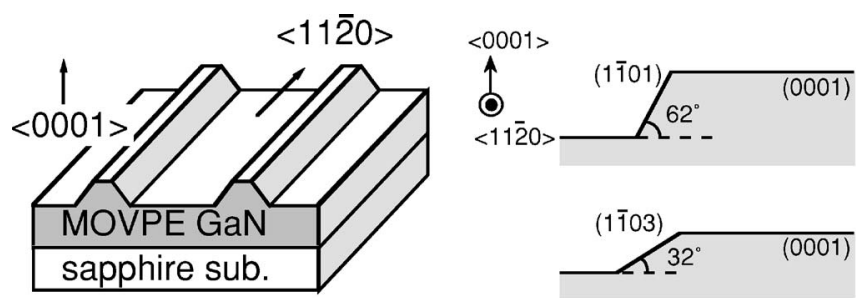

(a)

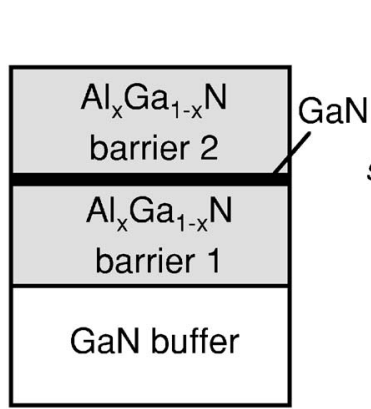

(b)

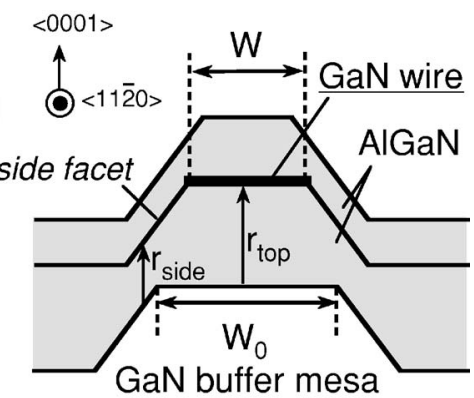

(c)
FIG. 1. Selective growth sequence used in this study. (a) Preparation of patterned substrates, (b) material supply, and (c) schematic illustration of the embedded GaN wire.

\section{SELECTIVE GROWTH EXPERIMENTS}

\section{A. Experimental method}

As templates for the selective growth, $\langle 11-20\rangle$-oriented mesa stripes were fabricated by etching of MOVPE grown GaN layer on (0001) sapphire substrates, as shown in Fig. 1(a). Etching was carried out by an electron cyclotron resonance assisted reactive ion beam etching (ECR-RIBE) process, developed for nitrides by our group. ${ }^{18}$ The ECR-RIBE process produced sidewalls corresponding to well-defined crystalline facets. These features of the templates are important for the well-defined and reproducible growth. In this study, two kinds of mesas having different side facets such as (1-101) facets and (1-103) facets were fabricated in the same $\langle 11-20\rangle$ direction, and they were employed to investigate the basic growth features.

Before loading into the MBE chamber, the template surfaces were cleaned by aceton and ethanol with ultrasonic agitation for $5 \mathrm{~min}$, and then subsequently treated in HF solution for $6 \mathrm{~min}$ in the atmosphere. After loading into the MBE chamber, thermal cleaning was applied for 5 min just prior to growth under the irradiation of the $\mathrm{N}_{2}$ radical excited with a microwave power of $350 \mathrm{~W}$ and the $\mathrm{N}_{2}$ flow rate of 0.5 SCCM (SCCM denotes cubic centimeter per minute at STP). From detailed observations of the reflective high energy electron diffraction (RHEED) patterns, cleaning at a substrate temperature around $800{ }^{\circ} \mathrm{C}$ was found to be effective in removing the native oxide or other contaminations from the surface.

A typical material supply and the schematic illustration of embedded GaN wires are shown in Figs. 1(b) and 1(c), respectively. Undoped GaN buffer layer was first grown on a patterned substrate. Then, the $\mathrm{AlGaN} / \mathrm{GaN}$ layers were

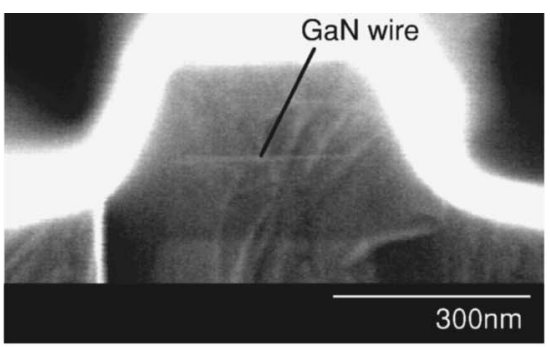

(a)

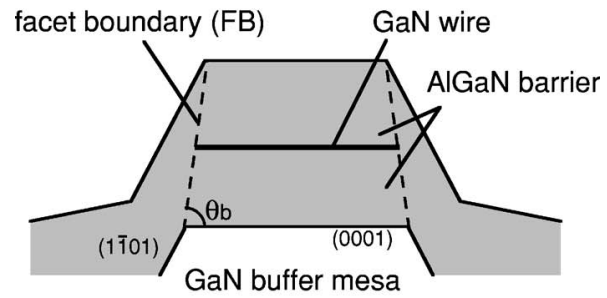

(b)

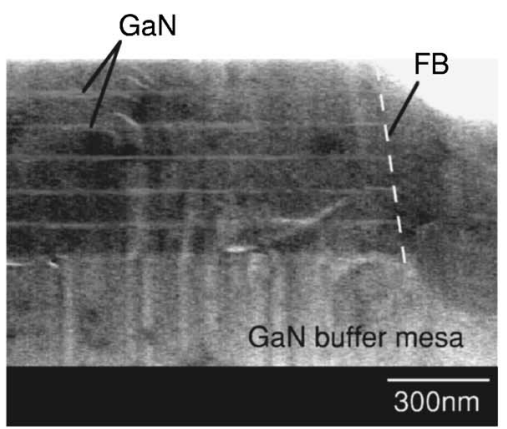

(c)

FIG. 2. (a) Cross-sectional SEM image of $\mathrm{GaN} / \mathrm{Al}_{0.24} \mathrm{Ga}_{0.76} \mathrm{~N}$ wire and (b) its schematic illustration. (c) Cross-sectional SEM image of the sample after repeated growth of $\mathrm{Al}_{0.24} \mathrm{Ga}_{0.76} \mathrm{~N}(100 \mathrm{~nm}) / \mathrm{GaN}(5 \mathrm{~nm})$ on basic one-sided mesa.

grown on the buffer mesa for the selective growth of $\mathrm{GaN}$ nanowires. In this study, the $\mathrm{Al}$ composition, $X$, in $\mathrm{Al}_{X} \mathrm{Ga}_{1-X} \mathrm{~N}$ layer was controlled from $0(\mathrm{GaN})$ to 0.3 by changing the $K$-cell temperature of $\mathrm{Al}$ source $T_{\mathrm{Al}}$ keeping that of Ga source $T_{\mathrm{Ga}}$ at $940{ }^{\circ} \mathrm{C}$.

In order to clarify the basic growth properties, the single $\mathrm{GaN}$ or $\mathrm{Al}_{X} \mathrm{Ga}_{1-X} \mathrm{~N}$ layers with a typical thickness of $100-300 \mathrm{~nm}$ were also grown on the wide mesas. The dislocation density of the grown AlGaN layers can be roughly estimated in $1-2 \times 10^{9} \mathrm{~cm}^{-2}$ from that of the GaN templates. By taking account of the growth thickness used in this study, the strain of $\mathrm{Al}_{X} \mathrm{Ga}_{1-X} \mathrm{~N}$ layers seems to be almost relaxed. ${ }^{19}$ The precise Al composition $X$ was determined by the x-ray diffraction (XRD) analysis on the sample grown on a planar (0001) substrate placed near the patterned substrate in the chamber. The $\mathrm{Al}$ composition dependence on the various features of selective growth was investigated by the detailed cross-sectional observation using Hitachi S-4100 SEM.

\section{B. Cross sections of embedded wires and facet boundaries}

Figures 2(a) and 2(b) show the cross-sectional SEM im- 


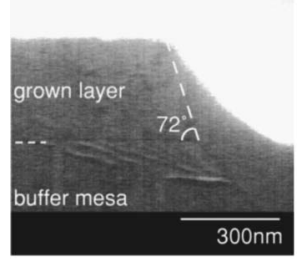

(a)

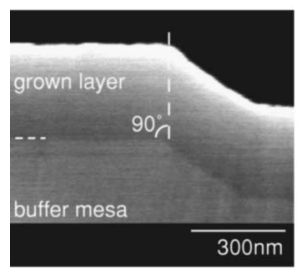

(c)

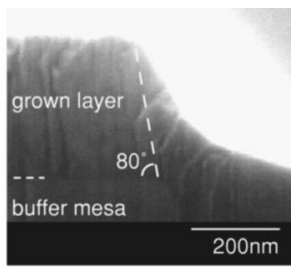

(b)

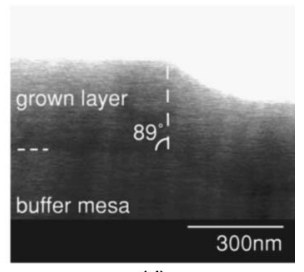

(d)
FIG. 3. Cross-sectional SEM images of the sample grown by changing $\mathrm{Al}$ composition: (a) GaN layer and (b) $\mathrm{Al}_{0.24} \mathrm{Ga}_{0.76} \mathrm{~N}$ layer grown on the mesa with (1-101) side facet. (c) $\mathrm{Al}_{0.11} \mathrm{Ga}_{0.89} \mathrm{~N}$ layer and (d) $\mathrm{Al}_{0.24} \mathrm{Ga}_{0.76} \mathrm{~N}$ layer grown on the mesa with (1-103) side facet.

age and its schematic illustration of the sample after the growth of $\mathrm{Al}_{0.24} \mathrm{Ga}_{0.76} \mathrm{~N} / \mathrm{GaN} / \mathrm{Al}_{0.24} \mathrm{Ga}_{0.76} \mathrm{~N}$ layers on the $\mathrm{GaN}$ buffer mesa defined by top (0001) and side (1-101) facets. As seen in Fig. 2(a), the embedded $\mathrm{GaN}$ wire was selectively formed on the top (0001) plane of the AlGaN mesa with decreased lateral width.

To see how the wire width and position are determined, a series of repeated growth experiments were carried out on simple one-sided mesa steps. Resultant cross-sectional SEM image of the sample is shown in Fig. 2(c), where the repeated growth of $\mathrm{Al}_{0.24} \mathrm{Ga}_{0.76} \mathrm{~N}(100 \mathrm{~nm}) / \mathrm{GaN}(5 \mathrm{~nm})$ layers was carried out by using GaN layers as markers. As shown in Fig. 2(c), the boundary separating the growth region between the top (0001) and the side (1-101) facets can be clearly identified after repeated growth. We call this boundary the facet boundary (FB) as we did firstly for GaAs wires in our previous study. ${ }^{16}$ Apparently, two FBs determine the lateral size of the wires formed selectively on the mesa top, as schematically shown in Fig. 2(b). Thus, the facet boundary angle $\theta_{b}$, with respect to the flat (0001) plane is an important parameter in controlling the wire width by the present selective growth technique.

\section{Dependences of cross-sectional features on Al composition}

In order to further clarify the evolution mechanism of the cross-sectional features, the growth experiments were carried out by changing the $\mathrm{Al}$ composition $X$ in $\mathrm{Al}_{X} \mathrm{Ga}_{1-X} \mathrm{~N}$ layer on two kinds of initial mesas having different side facets schematically shown in the right of Fig. 1(a). Figures 3(a) and 3(b) show the cross-sectional SEM images of the sample after growth of a single $\mathrm{GaN}(X=0)$ layer and an $\mathrm{Al}_{0.24} \mathrm{Ga}_{0.76} \mathrm{~N}$ layer on the one-sided mesa having the (1-101) side facet, respectively. In the cross sections of the single layer grown without any periodical markers, the facet boundaries might not be visible. As shown in Figs. 3, the facet boundaries were indicated by the dashed lines as eye guides,

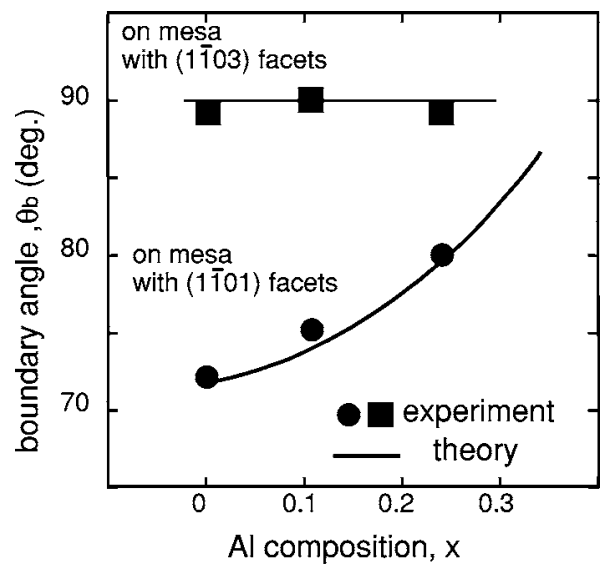

(a)

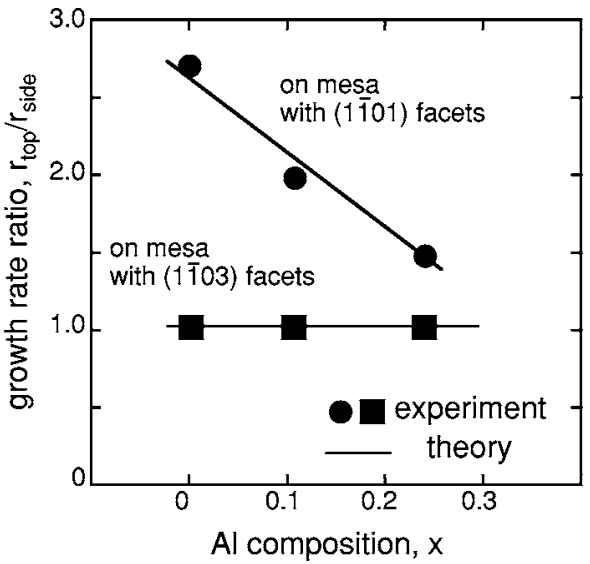

(b)

FIG. 4. Al composition dependences of the growth features for (a) facet boundary angle $\theta_{b}$ and (b) the growth rate ratio $r_{\text {top }} / r_{\text {side }}$.

on the basis of the fact that facet boundary are defined by the planes separating the growth regions between the top and the side facets. As shown in Figs. 3(a) and 3(b), it was found that the angle of facet boundaries $\theta_{b}$ depends on the $\mathrm{Al}$ composition.

On the other hand, in the case of the growth on the mesa having the (1-103) side facet, the boundary angle did not change even if the Al composition $X$ changed. Figures 3(c) and 3(d) show the cross-sectional SEM images of the sample after growth of a single $\mathrm{Al}_{0.11} \mathrm{Ga}_{0.89} \mathrm{~N}$ layer and an $\mathrm{Al}_{0.24} \mathrm{Ga}_{0.76} \mathrm{~N}$ layer on the one-sided mesa having the (1-103) side facet, respectively. The boundary angle was approximately $90^{\circ}$ for both samples.

The facet boundary angles $\theta_{b}$ are plotted in Fig. 4(a) as a function of the $\mathrm{Al}$ composition $X$ for the samples grown on the two kinds of mesas defined by the different side facets. The solid lines in Fig. 4(a) are simulated values explained later. As for the growth on the mesa defined by the (1-101) side facets, it was found that the value of $\theta_{b}$ significantly changed form $72^{\circ}$ up to $80^{\circ}$ as $X$ increased from 0 to 0.24 . On the other hand, the value of $\theta_{b}$ did not change with $X$ in the case of the growth on the mesa defined by the (1-103) side facets, as shown in Fig. 4(a). Such complicated growth 
features are obviously correlated with the growth kinetics including the atom migration and incorporation on the grown surfaces.

From simple geometrical consideration, the boundary angle $\theta_{b}$ should satisfy the following relation, as derived previously for GaAs/AlGaAs wires ${ }^{16}$ and GaN/AlGaN wires. ${ }^{15}$

$$
\tan \theta_{b}=\frac{\alpha \tan \theta_{\text {side }}-\tan \theta_{\text {top }}}{\alpha-1} .
$$

Here, $\alpha$ is the growth rate ratio defined as $\alpha=r_{\text {top }} / r_{\text {side }}$, where $r_{\text {top }}$ is the vertical growth rate on the top facet, and $r_{\text {side }}$ is that on the side facet, as defined in Fig. 1(c). $\theta_{\text {top }}$ and $\theta_{\text {side }}$ are the angles of top facet and side facet with respect to a flat top of mesa, respectively. In this study, the value of $\theta_{\text {top }}=0^{\circ}$ should be used as the angle of top (0001) facet. $\theta_{\text {side }}=62^{\circ}$ and $32^{\circ}$ should be used as the angle of side (1-101) facet and (1-103) facet, respectively.

Thus, another important parameter for the selectively grown wire is the growth selectivity on the top facet versus the growth on the side facets. Figure 4(b) compares the measured growth rate ratio, $\alpha=r_{\text {top }} / r_{\text {side, }}$ for the samples grown by changing the $\mathrm{Al}$ composition. The solid lines indicate the simulated values explained later.

For all samples grown on the mesa defined by the (1-101) side facets, the growth rate ratio $\alpha$ was larger than unity. This indicates that the incorporation rate of the adatoms on the top facet is larger than those on the side facets. It was also found that the value of $\alpha$ decreases as the Al composition is increased, as seen in Fig. 4(b). This is probably caused by two factors. Namely, one is that the incorporation rate of $\mathrm{Al}$ adatoms is much larger than that of $\mathrm{Ga}$ adatom, and another is that the migration length of $\mathrm{Al}$ adatoms is much shorter than that of Ga adatoms. From these factors, as the Al composition of AlGaN layer is increased, a total amount of $\mathrm{Al}$ adatom migrating from side facets to the top facet are decreased, leading to the poor selectivity.

On the other hand, the growth selectivity $\alpha$ on the mesa defined by the (1-103) side facets was almost unity and smaller than that on the mesa defined by the (1-101) side facet. This result suggests that the incorporation rate of adatoms on the (1-103) facet is larger than that on the (1-101) facets.

\section{COMPUTER SIMULATION OF SELECTIVE GROWTH}

\section{A. Basic equations}

For the quantitative theoretical description, a computer simulation method which our group recently developed for GaAs-based materials ${ }^{16,17}$ was applied here for GaN-based materials. This computer simulation was based on a phenomenological continuum growth model in which the growth process is described by a diffusion equation with macroscopic parameters such as diffusion constant and lifetime of adsorbed adatoms on growing surfaces.
In this modeling, the surface density of group III adatoms, $n\left(x, t_{g}\right)$, at the lateral position $x$ and the growth time $t_{g}$ are assumed to satisfy the following phenomenological equations:

$$
\begin{aligned}
& \frac{d n\left(x, t_{g}\right)}{d t_{g}}=G \cos \theta-\frac{n\left(x, t_{g}\right)}{\tau(\theta)}-\frac{d J\left(x, t_{g}\right)}{d x}, \\
& J\left(x, t_{g}\right)=-D \frac{n\left(x, t_{g}\right)}{k_{B} T} \operatorname{grad}(U),
\end{aligned}
$$

where $n$ is the adatom density, $G$ is the incoming molecular beam flux, and $J$ is the surface diffusion flux of adatoms. $\tau(\theta)$ denotes the average lifetime of group III atoms in adatom phase at the surface and it is given by

$$
1 / \tau(\theta)=1 / \tau_{i}(\theta)+1 / \tau_{d}(\theta),
$$

where $\tau_{i}(\theta)$ and $\tau_{d}(\theta)$ are the lifetime until incorporation into the crystalline facet and that until desorption from the surface to vacuum, respectively. Here, $\theta$ is the angle of the slope of the growing surface at $x$ with respect to the (0001) plane, and we assume that the lifetime of adatoms depends strongly on the surface slope $\theta$. In fact, the $\theta$ dependence of $\tau(\theta)$ gives the growth selectivity between the neighboring facets. $D$ and $U$ are the surface diffusion coefficient and chemical potential of adatoms on a facet, respectively.

After the calculation of the adatom density, $n\left(x, t_{g}\right)$ as a function of growth time $t_{g}$, the cross-sectional growth profile is obtained by plotting the vertical growth thickness $T\left(x, t_{g}\right)$, which is represented by the following equation, as a function of lateral position $x$ and growth time $t_{g}$ :

$$
T\left(x, t_{g}\right)=\int_{0}^{t_{g}} \frac{n(x, t) \Omega}{\tau_{i}(\theta) \cos \theta} d t .
$$

When there are more than one species of group III adatoms as in the case of growth of AlGaN, Eq. (2) was solved separately for each species, and each contribution was added together in calculating Eq. (5).

\section{B. Determination of parameter values for simulation}

It is obvious that the surface lifetime $\tau$ and the diffusion coefficient $D$ of group III adatoms in Eqs. (2) and (3) are the two important parameters that determine the growth features. In our previous work for GaAs-based materials, ${ }^{16,17}$ we have assumed that the lifetime of adatoms for the desorption $\tau_{d}(\theta)$ is much larger than that for the incorporation $\tau_{i}(\theta)$ where the average lifetime $\tau(\theta)$, denoted in Eq. (4), is regarded as to be same with $\tau_{i}(\theta)$. This assumption seems to be satisfactory for the growth of GaAs-based materials in the group III limited mode under the high As pressure. However, it is not the case for the growth of GaN in the group V supply limited mode, as reported in Ref. 15 where the incorporation rate of $\mathrm{Ga}$ adatoms was approximately half of the Ga supply flux from the $K$-cell source. Therefore, it was assumed that $\tau_{i}(\theta): \tau_{d}(\theta)=1: 1$ for $\mathrm{Ga}$ adatoms in this study, whereas the terms of $\tau_{d}(\theta)$ for $\mathrm{Al}$ adatoms was ignored due to the high incorporation rate of $\mathrm{Al}$ adatoms. 
As for the slope dependent lifetime until incorporation $\tau_{i}(\theta)$, it has been shown that the following theoretical formula obtained by Ohtsuka and Miyazawa ${ }^{20}$ and Ohtsuka ${ }^{21}$ gives satisfactory results in reproducing experiments for GaAs-based materials. ${ }^{17}$ These equations were derived by solving a diffusion equation on a terrace with steps. Therefore, they are basically applicable here for GaN-based materials.

$$
\begin{aligned}
& \tau_{i}(\theta)=\left[1-\alpha_{\text {step }}(\theta)\right] \tau_{i}(0), \\
& \alpha_{\text {step }}(\theta)=\frac{\lambda_{0} / \delta_{ \pm}}{1+\left(\lambda_{0} / \delta_{ \pm}\right) \operatorname{coth}\left[L(\theta) / 2 \lambda_{0}\right]} \frac{2 \lambda_{0}}{L(\theta)},
\end{aligned}
$$

where $\tau(0)$ is the lifetime of adatoms at the singular surface appeared during the selective growth process. $\lambda_{0}$ is the diffusion length of adatoms defined as $\lambda_{0}=[\tau(0) D]^{1 / 2}$ using the diffusion coefficient $D$. The $\delta \pm$ is the parameter related to the step densities.

To use the above equations, we have to know which facets appear during growth, and what are the values of $\tau_{i}(0)$ on the singular surfaces of these facets. From the repeated growth experiments on patterned substrates, we assumed that the growth on (0001) patterned substrates was characterized by the appearance of specific facets of the (1-101) and (1-103) planes in addition to the initial (0001) plane. This assumption seems to be consistent with the result that the (1-101) and (1-103) facets were easy to appear on the (0001) GaN substrate by the etching process using the ECR-RIBE technique. Here, it was also assumed that the growth rate ratio measured in Fig. 4(b) corresponds to the lifetime ratio at specific facets. For example, the relations of lifetimes, $\tau_{0001}, \tau_{1-103}$, and $\tau_{1-101}$, respectively, on (0001), (1-103), and (1-101) planes were set to be $\tau_{0001}: \tau_{1-103}: \tau_{1-101}=1: 1: 2.7$ for Ga adatoms.

As for the diffusion constant, it can be assumed that $D$ is strongly dependent on the temperature $T_{\text {sub }}$ as in the following equation:

$$
D=D_{0} \exp \left(-E_{d} / k_{B} T_{\text {sub }}\right),
$$

where, $E_{d}$ is the activation energy of surface adatoms diffusion. In this study, $D_{0}=2.4 \times 10^{-5} \mathrm{~m}^{2} / \mathrm{s}$ and $E_{d}=1.4 \mathrm{eV}$ were used for the growth of $\mathrm{GaN}$ on the (0001) plane, referring to the works by Liu et al. ${ }^{22}$ and Mao et al. ${ }^{23}$ The value of $D$ was set to be $6.2 \times 10^{-12} \mathrm{~m}^{2} / \mathrm{s}$ at $T_{\text {sub }}=800^{\circ}$ for Ga adatoms. Furthermore, in order to take account of the migration length difference in $\mathrm{Ga}$ and $\mathrm{Al}$ atoms, we assumed that the value of $D$ for Al adatoms is 100 times smaller that that for Ga adatoms, i.e., $\lambda_{0} \mathrm{Ga}\left(=10 \lambda_{0} \mathrm{Al}\right)$, in the calculation.

\section{Reproduction of growth experiments by simulation}

By using the above relation of the diffusion coefficient $D$ as well as the values of the lifetime mentioned previously, the growth profiles of $\mathrm{AlGaN}$ layer on the GaN buffer mesa were calculated. Figures 5(a) and 5(b) show the growth profiles calculated for the $\mathrm{Al}_{0.24} \mathrm{Ga}_{0.76} \mathrm{~N}$ layers grown on the GaN buffer mesa having the initial mesa width $\mathrm{W}_{0}$ of 300 and $50 \mathrm{~nm}$, respectively. Here, the case was considered that the GaN buffer mesas were defined by the top (0001) and

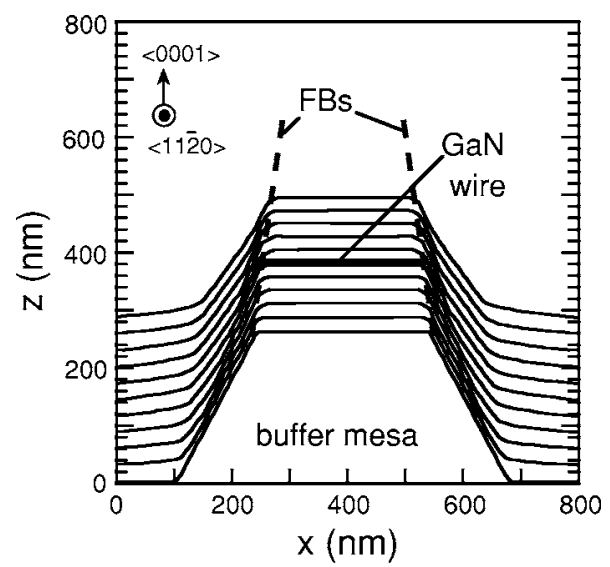

(a)

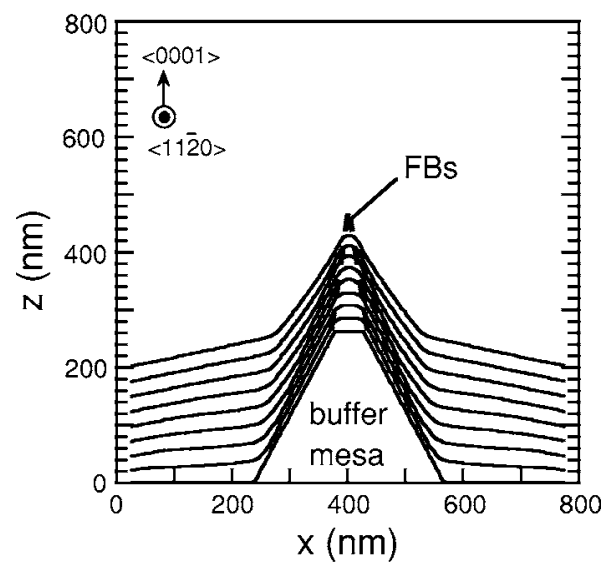

(b)

FIG. 5. Calculated growth profiles of AlGaN layers on (a) wide mesa with a top size of $300 \mathrm{~nm}$ and (b) narrow mesa with a top size of $50 \mathrm{~nm}$. The bold line drawn on the growth profile in Fig. 5(a) corresponds to the position of GaN wire of the sample shown in Fig. 2(a).

side (1-101) facets. The present simulation well reproduced the growth features on nonplanar substrates by setting the actual growth parameters in the calculation. For example, the cross-sectional features of the experimentally grown $\mathrm{GaN}$ wire seen in Fig. 2(a) were well reproduced by the simulation, as shown in Fig. 5(a), not only for the lateral wire width but also for the vertical wire position. Furthermore, the calculation results for the boundary angle $\theta_{b}$ and the growth rate ratio $r_{\text {top }} / r_{\text {side }}$ were very consistent with the experimental data obtained by changing the Al composition, as shown in the solid lines in Figs. 4(a) and 4(b). From the simulation results on the narrower mesa shown in Fig. 5(b), the further reduction of lateral wire width is expected.

Figure 6 shows the measured top mesa width $W$ of the samples grown on the GaN buffer mesas having the initial width $\mathrm{W}_{0}$ of $500 \mathrm{~nm}$ for a fixed $\mathrm{Al}$ composition of $X=0.24$. The solid line in Fig. 6 shows the calculated results. The theoretical curve obtained in the present simulation showed good agreements with the experimental data. These results indicate that the lateral width of present embedded wires can 


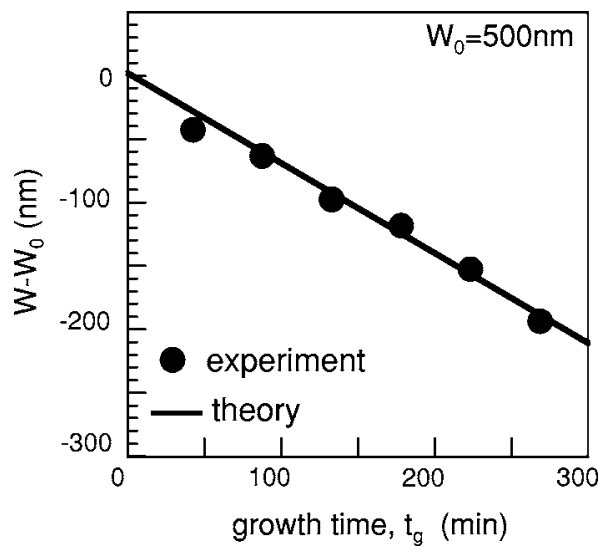

FIG. 6. Experimental and theoretical values of the lateral wire width plotted as functions of the growth time of $\mathrm{AlGaN}$ barrier layers.

be kinetically controlled by the growth conditions and the growth time of the $\mathrm{AlGaN}$ layer prior to the start of the wire growth.

\section{CONCLUSION}

In this study, the mechanism of the cross-sectional evolution during the selective MBE growth of $\langle 1-120\rangle$-oriented GaN wires was studied both experimentally and theoretically. From the detail investigation on the growth profiles, it was found that the lateral wire width is determined by two facet boundaries formed within AlGaN layers. The growth selectivity and the measured angle of facet boundary strongly depended on the $\mathrm{Al}$ composition in $\mathrm{Al}_{X} \mathrm{Ga}_{1-X} \mathrm{~N}$ layers and the crystalline facets of the initial mesa templates. Detailed features of cross-sectional structures could be well reproduced by a computer simulation based on a phenomenological continuum growth model where the slope angle dependence of lifetime of adatoms was taken into account. It is concluded that the lateral width of present nanowires can be kinetically controlled by the growth conditions and the supply thickness of AlGaN layers.

\section{ACKNOWLEDGMENTS}

The work reported here is supported in part by the 21st Century COE Project at Hokkaido University on "MemeMedia Technology Approach to the R\&D of Next-Generation Information Technologies," by a Grant-in-Aid for Young Scientists (B) under Grant No. 16760239, and by Grant-in-Aid for Exploratory Research through Grant No. 17656099, all from the Japanese Government.

${ }^{1}$ M. Miyamura, K. Tachibana, T. Someya, and Y. Arakawa, J. Cryst. Growth 237-239, 1316 (2002).

${ }^{2}$ J. Brown, F. Wu, P. M. Petroff, and J. S. Speck, Appl. Phys. Lett. 84, 690 (2004).

${ }^{3}$ H. Hirayama, S. Tanaka, P. Ramvall, and Y. Aoyagi, Appl. Phys. Lett. 72, 1736 (1998).

${ }^{4}$ S. Tanaka, M. Takeuchi, and Y. Aoyagi, Jpn. J. Appl. Phys., Part 2 39, L831 (2000).

${ }^{5}$ M. Yoshizawa, A. Kikuchi, M. Mori, N. Fujita, and K. Kishino, Jpn. J. Appl. Phys., Part 2 36, L459 (1997).

${ }^{6}$ J. R. Kim, H. M. So, J. W. Park, J. J. Kim, J. Kim, C. J. Lee, and S. C. Lyu, Appl. Phys. Lett. 80, 3548 (2002).

${ }^{7}$ E. Kapon, D. M. Hwang, and R. Bhat, Phys. Rev. Lett. 63, 430 (1989).

${ }^{8}$ T. Fukui, S. Ando, Y. Tokura, and T. Toriyama, Appl. Phys. Lett. 58, 2018 (1991).

${ }^{9}$ S. Koshiba et al., Appl. Phys. Lett. 64, 363 (1994).

${ }^{10}$ X. L. Wang, M. Ogura, and H. Matsuhata, Appl. Phys. Lett. 66, 1506 (1995).

${ }^{11}$ H. Fujikura and H. Hasegawa, J. Electron. Mater. 25, 619 (1996).

${ }^{12}$ T. Sato, I. Tamai, C. Jiang, and H. Hasegawa, Inst. Phys. Conf. Ser. 170-4, 325 (2002).

${ }^{13}$ I. Tamai, T. Sato, H. Hasegawa, and T. Hashizume, e-J. Surf. Sci. Nanotechnol. 4, 19 (2006).

${ }^{14}$ T. Oikawa, F. Ishikawa, T. Sato, T. Hashizume, and H. Hasegawa, Appl. Surf. Sci. 244, 84 (2005).

${ }^{15}$ T. Sato, T. Oikawa, and H. Hasegawa, Jpn. J. Appl. Phys., Part 1 44, 2487 (2005).

${ }^{16}$ T. Sato, I. Tamai, and H. Hasegawa, J. Vac. Sci. Technol. B 22, 2266 (2004).

${ }^{17}$ T. Sato, I. Tamai, and H. Hasegawa, J. Vac. Sci. Technol. B 23, 1706 (2005).

${ }^{18}$ H. Hasegawa, T. Muranaka, S. Kasai, and T. Hashizume, Jpn. J. Appl. Phys., Part 1 42, 2375 (2003).

${ }^{19}$ J. Qu, J. Li, and G. Zhang, Solid State Commun. 107, 467 (1998).

${ }^{20}$ M. Ohtsuka and S. Miyazawa, J. Appl. Phys. 64, 3522 (1988).

${ }^{21}$ M. Ohtsuka, J. Cryst. Growth 205, 112 (1999).

${ }^{22}$ H. Liu, J. G. Kim, M. H. Ludwig, and R. M. Park, Appl. Phys. Lett. 71, 347 (1997).

${ }^{23}$ H. Mao, S. Lee, and S. J. Park, Surf. Sci. 432, L617 (1999). 